This paper is a preprint of a paper submitted to IET Generation, Transmission \& Distribution. If accepted, the copy of record will be available at the IET Digital Library.

\title{
An Efficient Adaptive Virtual Inertia Controller for Virtual Synchronous Generators
}

\author{
Mostafa Malekpour ${ }^{1}$, Arash Kiyoumarsi ${ }^{1}$, Mehdi Gholipour ${ }^{1}$ \\ ${ }^{1}$ Department of Electrical Engineering, Faculty of Engineering, University of Isfahan, Isfahan, Iran 81746-73441 \\ $\bowtie$ E-mail: kiyoumarsi@eng.ui.ac.ir
}

\begin{abstract}
This paper proposes an efficient adaptive strategy to control virtual inertia of virtual synchronous generators (VSGs). This adaptive virtual inertia can provide low frequency oscillation (LFO) damping and simultaneously improve primary frequency control (PFC) in power systems. The virtual inertia is synthesized by two components named the LFO and the PFC components. The former provides LFO damping. It increases the virtual inertia whenever the VSG frequency diverges from grid center-of-inertia (COI) frequency and decreases it when the VSG frequency converges to the COI frequency. The PFC component increases the virtual inertia after a power outage event until the COI frequency reaches its nadir in order to reduce the rate of grid frequency drop. Following frequency nadir instant, the PFC component decreases the virtual inertia to achieve a faster frequency recovery. The efficiency of the suggested method is validated through a power system with VSG penetration level ranges from $25 \%$ to $75 \%$ of the system generation rating. The achieved results verify the superiority of the proposed scheme in the PFC improving and the LFO damping in comparison with the existing methods. Moreover, the suggested approach decreases the required size of the VSG energy storage by about $15 \%$.
\end{abstract}

\section{Introduction}

Inertia of a power system is defined as its ability to oppose deviations in system frequency due to resistance provided by kinetic energy of rotating shafts in turbinegenerators [1]. The rate-of-change-of-frequency $(\mathrm{RoCoF})$ is inversely proportional to the system inertia [2]. It is believed that converter-based devices may constitute more than $50 \%$ of the rated capacity of future networks [3]. These devises do not inherently provide inertia to the system and then system inertia will be radically reduced [4]. Virtual synchronous generator (VSG) concept will take over the responsibility of inertia support in the converter-dominated grids [5]. By deploying this control scheme, power converters can emulate the static and dynamic features of synchronous generators (SGs) [6].

Primary frequency control (PFC) aims to maintain a balance between system generation and its demand, using turbine governors [7]. In this context, the system frequency should not fall below a given value to avoid load shedding. Thus, system frequency RoCoF should be restricted to a specified value to buy time for starting the PFC action [8]. In the other hand, sufficient damping of system low frequency oscillations (LFOs) must be available to ensure a stable system operation [9]. However, the converterdominated grids may affect the PFC proficiency and the LFO damping. To solve this problem, parameters of the VSGs can be set freely to improve stability of the low inertia power systems [10].

In the case of the LFO damping, there are several offline [11]-[16] and on-line [17]-[22] techniques set forth in the literature to control the virtual inertia and damping coefficient of the VSGs. In the off-line methods, the VSG parameters are determined at the design stage. It was shown that the desirable speed response of the VSG power could not be achieved by only adjusting the VSG virtual inertia [11]. To tackle this issue, the damping coefficient of the VSG can be modified through a correction loop based on the derivative of the VSG torque [11]. However, damping coefficient variation affects the VSG power dynamics distinguishably under discrepant operating conditions [12]. Therefore, the suggested method in [11] was improved to derive the virtual inertia and damping coefficient of the VSG according to specified mode of its power control loop [12]. The VSG concept has been extended to control voltage source converters (VSCs) in high-voltage direct current (HVDC) transmission systems [13]. It was revealed that phase-locked loop (PLL) of the VSG-based HVDC has a negative impact on its LFO damping [14]. This negative effect can be eliminated by a phase compensation to change the phase of the PLL equivalent torque [14]. An enhanced power system stabilizer (PSS) was proposed for the VSGs in [15]. The suggested PSS improves the LFO damping of the VSG without scarifying its inertial response. In this PSS, the VSG frequency deviation with respect to the grid frequency was estimated by derivative of a trigonometric function of the VSG output voltage [15]. The VSG parameters setting should avoid modal proximity of the VSG to the LFO mode of the power systems. Otherwise, the VSGs will have detrimental impact on the LFO damping [16].

In the on-line methods, the VSG parameters can be modified even during system operation. In [17], the LFO damping was improved by appending a reactive powerdependent term into the VSG swing equation. In the added term, a factor was continuously changed using linearized deviations of the VSG active and reactive powers [17]. A bang-bang strategy for the VSG virtual inertia control was introduced in [18]. In this scheme, a big virtual inertia was selected for the VSG when its frequency diverges from the grid frequency. On the other hand, a small virtual inertia was adopted while the VSG frequency converges to the grid frequency. In [19], a self-adaptive scheme controls the virtual inertia and damping coefficient of the VSG. In this scheme, the virtual inertia is increased during the VSG frequency divergence interval, while the damping coefficient is fixed. On the contrary, the damping coefficient 
is increased in the VSG frequency convergence interval, whereas the virtual inertia remains constant [19]. Another adaptive strategy by selecting different virtual inertia and damping coefficient during distinguished operating states was established in [20]. Compared to the methods in [17][19], the optimal damping ratio for the VSG can be maintained, while fast speed response and small overshoot can also be assured [20]. To improve frequency regulation of microgrids, a unified expression was presented for the VSG virtual inertia [21]. In particular, the virtual inertia was built by two terms of fixed and variable values. The latter one was obtained by multiplying a fixed gain, the VSG frequency deviation and its time derivative [21]. Recently, a dual-adaptivity inertia control has been presented to optimize both the power and frequency oscillations of the VSGs [22]. This strategy decreases the virtual inertia when the VSG power deviation is large. It decreases the virtual inertia whenever the VSG frequency deviation is large.

In the case of the PFC proficiency, the VSG-based HVDC systems can be equipped with PFC [23] and automatic generation control [24] capabilities by adjusting their DC link voltage. For microgrids, an extended VSG (EVSG) was presented through combining the virtual inertia, virtual primary and secondary frequency controllers [25]. The parameters of the EVSG were optimally tuned using $H_{\infty}$ robust control method considering power fluctuations of the renewable energy sources and the system demand.

In the all described strategies, the VSG parameters setting to improve the LFO damping and the PFC performance was individually done so far. However, this paper proposes an efficient strategy to control parameters of the VSG in order to meet these objectives, simultaneously. In this regard, the VSG virtual inertia is synthesized by two parts named the PFC and the LFO components. To reduce system frequency gradient, the PFC component increases the virtual inertia when the system center-of-inertia (COI) frequency decreases. On the other hand, this component decreases virtual inertia during the COI frequency rebound period to achieve a faster frequency recovery. The LFO component increases the virtual inertia whenever the VSG frequency diverges from the COI frequency and vice versa. In this context, calculation of the VSG frequency deviation with respect to the COI frequency plays an important role. This study deploys an estimation of the second derivative of the VSG frequency to calculate this frequency deviation. The simulation results demonstrated the superiority of the proposed strategy in the system performance modification in comparison with the existing methods. Further, an energy storage is typically used in the VSG systems to emulate the inertia. The suggested approach decreases the capacity of this storage by about $15 \%$.

This paper is organized as follows: Section 2 elaborates on the equations of the VSG model. The proposed VSG virtual inertia control strategy is presented in Section 3 following the brief descriptions of two existing methods. The effectiveness of the suggested technique is evaluated in Section 4. Finally, the conclusions and further remarks are provided in Section 5.

\section{Virtual Synchronous Generator Model}

The different methods to model a VSG can be found in literature such as [26]. In this section, a fourth order model is established for the VSG by considering a round rotor synchronous generator. This model has a virtual field circuit in direct axis of its virtual rotor as shown in Fig. 1. It has also a virtual damper circuit in quadrature axis of the virtual rotor. These virtual circuits are denoted by $f d$ and $1 q$ subscripts, respectively. Time derivatives of flux linkages per second of these circuits can be extracted as follows [27]:

$\frac{d \psi_{f d}}{d t}=\omega_{n}\left(\frac{r_{f d}}{x_{a d}} v_{e}-r_{f d} i_{f d}\right), \quad \frac{d \psi_{1 q}}{d t}=-\omega_{n} r_{1 q} i_{1 q}$

where, $r_{f d}$ and $r_{1 q}$ are resistances of the field and damper circuits, respectively. Denote $\omega_{n}$ the nominal angular speed of the VSG. The field voltage is $v_{e}$ and the d-and q-axes magnetizing reactances $\left(x_{a d}\right.$ and $\left.x_{a q}\right)$ are defined as:

$x_{a d}=x_{d}-x_{l}, \quad x_{a q}=x_{q}-x_{l}$

with $x_{d}$ and $x_{q}$ as the $\mathrm{d}$ - and q-axes synchronous reactances. The leakage reactance of the virtual stator is denoted by $x_{l}$. The currents of the virtual rotor circuits $\left(i_{f d}\right.$ and $i_{1 q}$ ) can be expressed as [28]:

$i_{f d}=k_{f d} i_{d}+\frac{\psi_{f d}}{x_{f d}+x_{a d}} \quad i_{1 q}=k_{1 q} i_{q}+\frac{\psi_{1 q}}{x_{1 q}+x_{a q}}$

where:

$k_{f d}=\frac{x_{a d}}{x_{f d}+x_{a d}} \quad k_{1 q}=\frac{x_{a q}}{x_{1 q}+x_{a q}}$

Reactances of the virtual field and damper windings are represented by $x_{f d}$ and $x_{1 q}$, correspondingly. The d- and qaxes magnetizing flux linkages per second $\left(\psi_{a d}\right.$ and $\left.\psi_{a q}\right)$ in terms of currents are:

$\psi_{a d}=x_{a d}\left(-i_{d}+i_{f d}\right)$

$\psi_{a q}=x_{a q}\left(-i_{q}+i_{1 q}\right)$

The VSG virtual stator flux linkages become:

$\psi_{d}=-x_{l} i_{d}+\psi_{a d}$

$\psi_{q}=-x_{l} i_{q}+\psi_{a q}$

The virtual stator voltages $\left(u_{d}\right.$ and $\left.u_{q}\right)$ can be written as follows [9]:

$u_{d}=u_{d}^{\prime}+\omega x_{q}^{\prime} i_{q}$

$u_{q}=u_{q}^{\prime}-\omega x_{d}^{\prime} i_{d}$

where, the transient voltages are defined by [9]:

$u_{d}^{\prime}=-\omega \psi_{q}^{\prime}$

$u_{q}^{\prime}=\omega \psi_{d}^{\prime}$

with $\omega$ as the VSG rotor speed (or frequency). The transient flux linkages can be calculated as follows [28]:

$\psi_{d}^{\prime}=k_{f d} \psi_{f d}$

$\psi_{q}^{\prime}=k_{1 q} \psi_{1 q}$

The transient reactances are [27]:

$x_{d}^{\prime}=x_{l}+\frac{x_{f d} x_{a d}}{x_{f d}+x_{a d}} \quad x_{q}^{\prime}=x_{l}+\frac{x_{1 q} x_{a q}}{x_{1 q}+x_{a q}}$

The VSG rotor resistances ( $r_{f d}$ and $r_{1 q}$ ) can be derived in
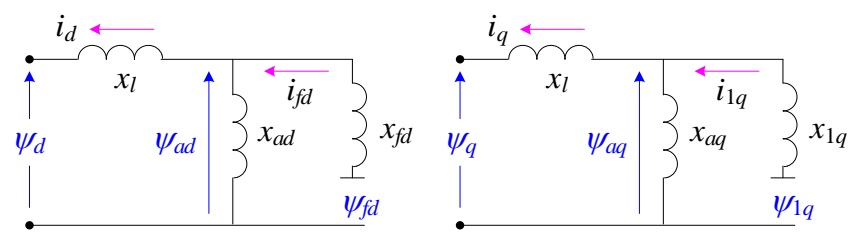

Fig. 1 The $d$-and q-axes equivalent circuits of the VSG model 
terms of the $\mathrm{d}$ - and q-axes open-circuit transient time constants $T_{d 0}^{\prime}$ and $T_{q 0}^{\prime}$ as follows [27]:

$$
r_{f d}=\frac{x_{f d}+x_{a d}}{\omega_{n} T_{d 0}^{\prime}} \quad r_{1 q}=\frac{x_{1 q}+x_{a q}}{\omega_{n} T_{q 0}^{\prime}}
$$

The VSG electromagnetic torque $T_{e}$ can be derived as follows [28]:

$T_{e}=\left(\psi_{d} i_{q}-\psi_{q} i_{d}\right) P F^{-1}$

where, $P F$ denotes nominal power factor of the VSG. The VSG electrical power $\left(P_{e}\right)$ is obtained by [27]:

$$
P_{e}=T_{e} \omega=u_{d} i_{d}+u_{q} i_{q}
$$

The VSG virtual rotor speed (or frequency) is governed by the swing equation as follows [28]:

$\frac{d}{d t} \omega=\frac{1}{T_{a g}}\left(\frac{P_{m}}{\omega}-T_{e}\right)$

with $P_{m}$ as virtual mechanical power. In (14), parameter $T_{a g}$ is virtual acceleration time constant, defined as [28]:

$T_{a g}=2 H\left(P F^{-1}\right)$

where, $H$ represents virtual inertia constant of the VSG in seconds [9]. The angle of the d-axis represented by $\varphi$, referenced to the real axis in stationary reference frame [27], can be determined as follows [28]:

$$
\frac{d}{d t} \varphi=\omega_{n}\left(\omega-\omega_{s l}\right)
$$

Denote $\omega_{s l}$ the slack machine frequency in per unit. Here, the q-axis leads the d-axis by 90 degrees.

The steady-state and transient saliencies of the VSG virtual rotor should be ignored to simplify its model. This means it can be assumed that $x_{d}=x_{q}$ and $x_{d}^{\prime}=x_{q}^{\prime}$. It is important to mention that dynamics of the VSG flux linkages will be deployed to estimate second time derivative of the VSG frequency. In addition, neglecting rotor saliencies in the VSG modelling results in more simplicity in the mentioned estimation. Note that the second-order VSG models in [18]-[22] do not have this capability. More details are provided in Section 3.4.

A VSG power plant may have supplementary elements as portrayed in Fig. 2. Denote $\bar{l}_{\alpha \beta}$ and $\bar{u}_{\alpha \beta}$ the VSG current and voltage vectors in the stationary reference frame [27]. An automatic voltage regulator (AVR) maintains the VSG voltage at the set point values. A power system stabilizer (PSS) may be used to provide additional damping torque [9]. The governor function is modelled by the primary controller (PCO) block. The virtual prime mover (VPM) simulates dynamics of a prime mover.

\section{Virtual Inertia Control Strategies}

In this section, two existing VSG virtual inertia control schemes proposed in [18] and [22] are firstly reviewed. Then, the proposed strategy is clearly presented in details.

\subsection{Bang-Bang Virtual Inertia Control Strategy}

In the bang-bang strategy, the virtual inertia of the VSG was modulated to provide the LFO damping as follows [18]:

$T_{a g}^{*}= \begin{cases}\left(1+k_{t p}\right) T_{a g 0} & \Delta \tilde{\omega}_{c} \frac{d}{d t} \omega>0 \\ \left(1+k_{t n}\right) T_{a g 0} & \Delta \tilde{\omega}_{c} \frac{d}{d t} \omega<0\end{cases}$

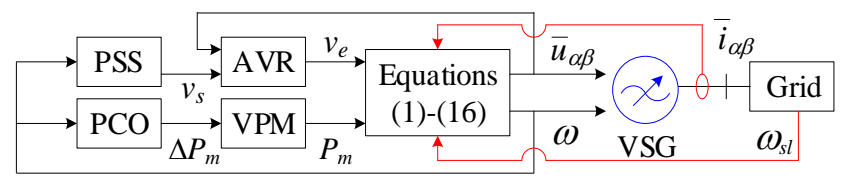

Fig. 2 Functional block diagram of the virtual synchronous generator plant

wherein, the virtual inertia increment and decrement with respect to the base virtual acceleration time constant $T_{a g 0}$, are determined by parameters $k_{t p}$ and $k_{t n}$, respectively. The feasible ranges of these parameters are as follows:

$k_{t p} \geq 0$

$-1<k_{t n} \leq 0$

Denote $\omega$ and $\Delta \widetilde{\omega}_{c}$ the VSG frequency and its deviation with respect to the grid frequency $\widetilde{\omega}_{c o i}[18]$ where:

$\Delta \tilde{\omega}_{c}=\omega-\tilde{\omega}_{c o i}$

Any method was not suggested in [18] to measure the grid frequency. In fact, it should be the COI grid frequency. However, the exact calculation of this quantity needs widearea measurements. As an alternative, an estimated COI frequency is used in this study, which is derived by moving average function as follows:

$\tilde{\omega}_{c o i}(t)=\frac{1}{T_{\text {avg }}} \int_{t-T_{\text {avg }}}^{t} \omega(t) d t$

with $T_{\text {avg }}$ as the time window of averaging. This parameter should be $50 \%$ greater than period of slowest low frequency oscillation in the power system. Note that the " $\sim$ " character is used in this paper to represent the estimated quantities.

\subsection{Dual-Adaptivity Virtual Inertia Control Strategy}

The dual-adaptivity virtual inertia control was proposed to mitigate the VSG frequency oscillations simultaneously with its power oscillations [22]. In this scheme, the virtual inertia of the VSG changes as follows:

$T_{a g}^{*}=\min \left(k_{t p}, \frac{k_{t n}+k_{t p} k_{a}^{2} \Delta \tilde{\omega}_{c}^{2}}{1+k_{a}^{2} \Delta \tilde{\omega}_{c}^{2}}\right)$

To achieve a compromise between frequency and power regulations, parameter $k_{a}$ was defined as [22]:

$k_{a}^{2}=\frac{k_{g} \Delta \tilde{\omega}_{c}^{2}}{1+\Delta \tilde{\omega}_{c}^{2}+\Delta P^{2}}$

where $k_{g}$ is a predesigned constant and $\Delta P$ can be written as: $\Delta P=P_{e}-P_{m}$

\subsection{Proposed Virtual Inertia Control Strategy}

For the sake of reliable operation of the VSG with adaptive virtual inertia, there is a need for energy storage, particularly when the VSG is deployed to control intermittent renewable energy sources. In this regard, the virtual inertia control efficiency is of vital consideration to minimize the capacity of the storage. In the other hand, flexibility in the virtual inertia has been used for providing the LFO damping and mitigating the PFC action individually so far. Indeed, this flexibility should be used to improve overall system performance. To this end, the VSG virtual inertia is defined as follows:

$T_{a g}^{*}=k_{p f c} T_{a g}^{p f c}+k_{l f o} T_{a g}^{l f o}$ 
wherein, the component $T_{a g}^{l f o}$ is used to improve the LFO damping while the component $T_{a g}^{p f c}$ is dedicated to the PFC mitigation. The participation of these components in the virtual inertia are given by the coefficients $k_{l f o}$ and $k_{p f c}$ which hold the following equality:

$k_{p f c}+k_{l f o}=1$

Before proceeding how these coefficients are calculated, let us consider derivation of the PFC and the LFO components. The former is derived as follows:

$T_{a g}^{p f c}=\left\{\begin{array}{cc}T_{a g 0}\left(1+k_{t p}\right) & k_{p f c}=1 \\ T_{a g}^{p f c 1} & k_{p f c}<1\end{array}\right.$

where, the auxiliary components $T_{a g}^{p f c 0}$ and $T_{a g}^{p f c 1}$ are defined as follows:

$T_{a g}^{p f c 0}= \begin{cases}\left(1+k_{t p}\right) T_{a g 0} & \Delta \tilde{\omega}_{c o i} \tilde{R}_{c o i}(t)>0 \\ \left(1+k_{t n}\right) T_{a g 0} & \Delta \tilde{\omega}_{c o i} \tilde{R}_{c o i}(t)<0\end{cases}$

$T_{a g}^{p f c 1}= \begin{cases}T_{a g 0} & \left|\tilde{R}_{c o i}(t)\right| \leq R_{\text {min }} \\ T_{a g}^{p f c 0} & \left|\tilde{R}_{c o i}(t)\right|>R_{\text {min }}\end{cases}$

where,

$\Delta \tilde{\omega}_{c o i}=\tilde{\omega}_{c o i}-1$

Do not confuse $\Delta \widetilde{\omega}_{c o i}$ and $\Delta \widetilde{\omega}_{c}$ defined in (19). The former represent deviation in the estimated COI frequency with respect to the system nominal frequency. Accordingly, an averaged time derivate of the $\widetilde{\omega}_{c o i}$ may be obtained as follows:

$$
\tilde{R}_{c o i}(t)=\frac{\tilde{\omega}_{c o i}(t)-\tilde{\omega}_{c o i}\left(t-T_{s p}\right)}{T_{s p}}
$$

in which, the sampling period $T_{s p}$ is set to $100 \mathrm{~ms}$ to comply with the commercially available RoCoF relays [29].

Fig. 3 illustrates how $T_{a g}^{p f c 1}$ is computed based on the estimated COI frequency and its time derivative. Hereinafter, all variables pertinent to $T_{a g}$ are plotted in per unit with $T_{a g 0}$ as the base value. When sign of the $\Delta \widetilde{\omega}_{\text {coi }}$ times its derivative is positive, i.e., the COI frequency is deviating from the nominal frequency, $T_{a g}^{p f c 1}$ is set to its upper limit and vice versa. However, $T_{a g}^{p f c 1}$ remains 1.0 p.u., whenever the absolute value of the COI frequency derivative $\tilde{R}_{c o i}$ is lower than a predefined value $R_{\min }$ (See eq. (28)). The PFC component $T_{a g}^{p f c}$ is identical with $T_{a g}^{p f c 1}$, except for $k_{p f c}$ lower than 1 . In the next page, it will be shown that $k_{p f c}$ remains 1.0 during one second after a frequency drop event. In other words, the component $T_{a g}^{p f c}$ aims at reducing COI

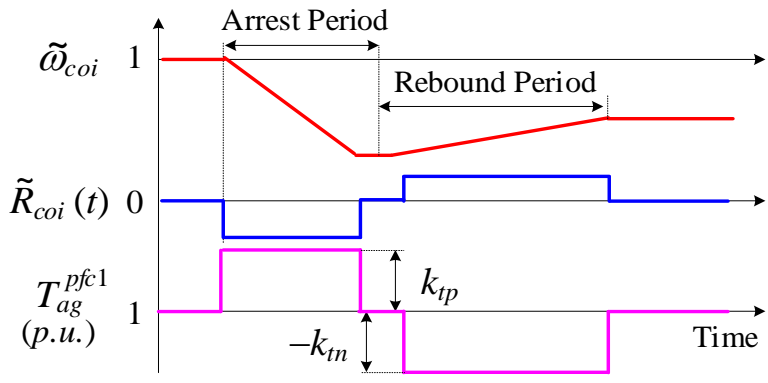

Fig. 3 The principle of the auxiliary PFC component $T_{a g}^{p f c 1}$ derivation

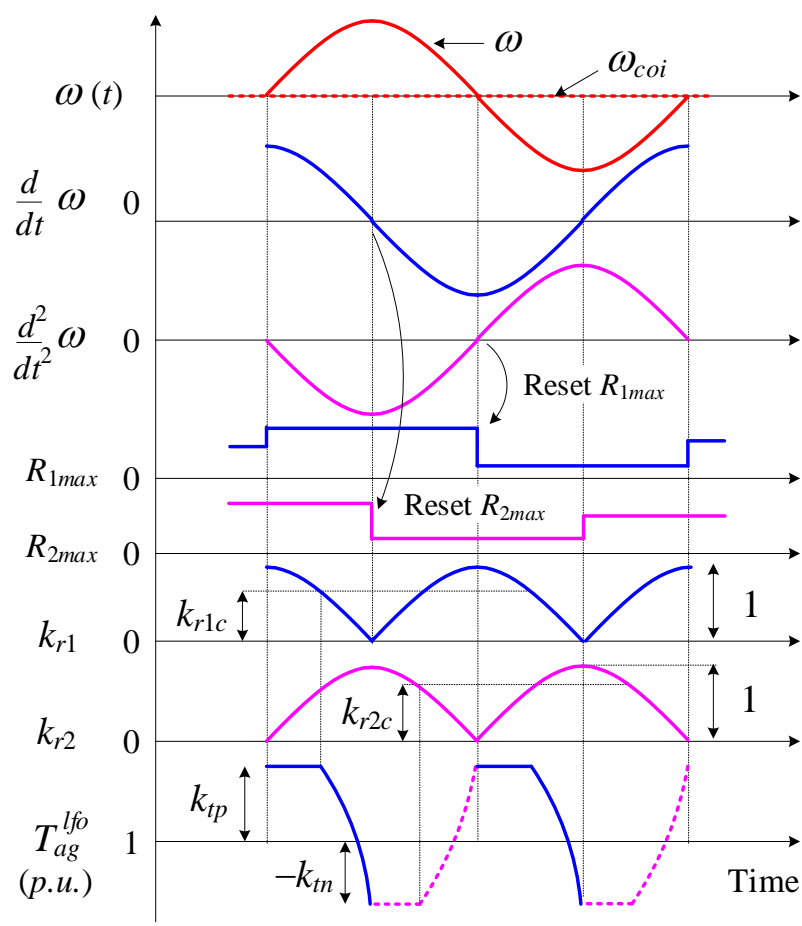

Fig. 4 The principles of the LFO component derivation

RoCoF during the frequency arrest period and increasing it at the frequency rebound stage.

The LFO component $T_{a g}^{l f o}$ is used to support the LFO damping. Thus, its operating frequency is similar with that of the LFO oscillations such as the bang-bang method [18]. In order to elucidate the principles of modulating this component, let us consider the VSG frequency $\omega$, which contains sinusoidal low frequency oscillations as portrayed in Fig. 4. The first and second time derivatives of the $\omega$ are also shwon. For off-nominal system frequencies, a major difficulty in providing the LFO damping by the bang-bang virtual inertia is the COI frequency estimation. Remember from (17) that, the sign of difference between the VSG frequency and the estimated COI frequency is employed to change the virtual inertia. In this regard, a strategy is proposed to derive this sign indirectly. As can be seen from Fig. 4, the sign of the second derivative of the $\omega$ has a negative value in contrast with $\Delta \widetilde{\omega}_{c}$, regardless of the COI frequency value. The second derivative may be calculated directly from the first derivative of the VSG frequency; however, another approach is deployed. The detail explanation of this approach is provided at Section 3.4.

Returning to Fig. 4, it can be observed that the $T_{a g}^{l f o}$ is modulated by a bang-bang method, which is modified through the variables $k_{r 1}$ and $k_{r 2}$. These variables are defined as:

$k_{r 1}=\left|\frac{d}{d t} \omega\right| R_{1 \max }^{-1} \quad k_{r 2}=\left|\frac{\tilde{d}^{2}}{d t^{2}} \omega\right| R_{2 \max }^{-1}$

where, $R_{1 \max }$ and $R_{2 \max }$ hold the maximum absolute values of the first and the second derivatives of the $\omega$ as:

$$
\begin{aligned}
& R_{1 \text { max }}=\max \left(\left|\frac{d}{d t} \omega\right|\right) \\
& R_{2 \max }=\max \left(\left|\frac{\tilde{d}^{2}}{d t^{2}} \omega\right|\right)
\end{aligned}
$$


The variables $R_{1 \max }$ and $R_{2 \max }$ are periodically reset at zero crossings of the second and first derivative of the $\omega$, respectively. Whenever the $\omega$ deviates from the $\omega_{\text {coi }}$, the variable $k_{r 1}$ gradually changes from 1 to zero. On the other hand, when the $\omega$ converges to the $\omega_{c o i}$, the variable $k_{r 2}$ gradually declines from 1 to zero. Accordingly, the LFO component $T_{a g}^{l f o}$ is determined as follows:

$$
T_{a g}^{l f o}= \begin{cases}T_{a g 0} \min \left(k_{t p}, k_{t n}+\left(k_{t p}-k_{t n}\right) \frac{k_{r 1}}{k_{r 1 c}}\right) & \left(\frac{\tilde{d}^{2} \omega}{d t^{2}}\right)\left(\frac{d \omega}{d t}\right)<0 \\ T_{a g 0} \max \left(k_{t n}, k_{t p}+\left(k_{t n}-k_{t p}\right) \frac{k_{r 2}}{k_{r 2 c}}\right) & \left(\frac{\tilde{d}^{2} \omega}{d t^{2}}\right)\left(\frac{d \omega}{d t}\right)>0\end{cases}
$$

where, parameters $k_{r 1 c}$ and $k_{r 2 c}$ are the predesigned constant ranges from zero to 1 . The solid parts of the $T_{a g}^{l f o}$ trace in Fig. 4 are determined by the first term of (33). In these sort of time intervals, the LFO component remains $k_{t p}$ until $k_{r 1}$ decreases to $k_{r 1 c}$. Subsequently, it slowly reduces to $k_{t n}$ with $k_{r 1}$. On the other hand, dotted parts of the $T_{a g}^{l f o}$ trace are modulated by the second term of (33). In this case, $T_{a g}^{l f o}$ maintains $k_{t n}$ until $k_{r 2}$ decreases to $k_{r 2 c}$. Afterwards, it softly increases to $k_{t p}$ with $k_{r 2}$. The proposed modified bang-bang virtual inertia $T_{a g}^{l f o}$ can be converted to that of defined by (17), if both critical constants $k_{r 1 c}$ and $k_{r 2 c}$ are set to zero. Nevertheless, the non-zero values are suggested in this study for these constants. In fact, the non-zero $k_{r 1 c}$ and $k_{r 2 c}$ moderate the VSG frequency derivative changes due to the virtual inertia changes. This moderation alleviates in turn the second derivative variations and gives a more precise zero-crossing detection for the VSG frequency derivatives.

The contribution of the $T_{a g}^{p f c}$ and $T_{a g}^{l f o}$ components in the virtual inertia can be determined by the $k_{p f c}$. This parameter may be defined as follows:

$$
k_{p f c}=\left\{\begin{array}{cl}
1 & f_{\text {evt }}=1 \\
k_{p f c 0}^{2}+\left(1-k_{p f c 0}\right) k_{p f c 1} & f_{\text {evt }}=0
\end{array}\right.
$$

where, the auxiliary PFC component participation factors $k_{p f c 0}$ and $k_{p f c 1}$ are illustrated in Fig. 5. Let the event flag $f_{\text {evt }}$ is initially zero. Suppose the system frequency abruptly drops at $t_{0}$. Next, the VSG increases its electrical power. Afterward, the flag $f_{\text {evt }}$ jumps to 1 when the power deviation $\Delta P$, defined in (23), reaches 0.01 per unit. The time $t_{\text {evt }}$ in Fig. 5 denotes this instant. Then, the flag $f_{\text {evt }}$ resets to zero after one second. The factor $k_{p f c 0}$ remains 1.0 during this time interval and then it reduces to zero within 2 seconds. In the other hand, the $k_{p f c 1}$ increases linearly with the absolute value of the COI RoCoF $\widetilde{R}_{c o i}$, defined by (30). The parameter $R_{\max }$ is the permissible maximum COI RoCoF [8]. Note that the second term in (34) is in fact the average value of the factors $k_{p f c 0}$ and $k_{p f c 1}$.
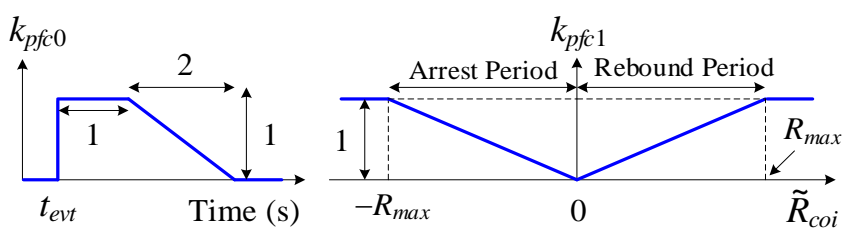

Fig. 5 The auxiliary participation factors of the PFC component

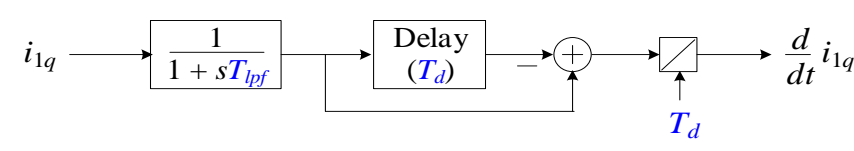

Fig. 6 Time derivative calculation for the VSG damper current

\subsection{Calculation of Second Derivative of the VSG Frequency}

From (14), ignoring time derivative of the virtual mechanical power $P_{m}$ in comparison with that of the electromagnetic torque, we have:

$\frac{\tilde{d}^{2}}{d t^{2}} \omega=-\frac{1}{T_{a g}} \frac{d}{d t} T_{e}=\frac{1}{T_{a g} P F} \frac{d}{d t}\left(\psi_{q} i_{d}-\psi_{d} i_{q}\right)$

The steady-state and transient non-saliencies in the VSG modelling allows us to reach the following expression for the second derivative of the VSG frequency:

$$
\begin{gathered}
\frac{\tilde{d}^{2}}{d t^{2}} \omega=\frac{1}{T_{a g} P F}\left(\left(i_{d}+\frac{\psi_{f d}}{x_{a d}}\right) \frac{d \psi_{1 q}}{d t}-\left(i_{q}+\frac{\psi_{1 q}}{x_{a d}}\right) \frac{d \psi_{f d}}{d t}\right. \\
\left.+\frac{1}{k_{f d}} \psi_{1 q} \frac{d}{d t} i_{f d}-\frac{1}{k_{f d}} \psi_{f d} \frac{d}{d t} i_{1 q}\right)
\end{gathered}
$$

where, the flux linkages derivatives are defined by (1). The current derivatives can be calculated by the delay and lowpass filter (LPF) functions as explained in Fig. 6. The time delay $T_{d}$ should be small in comparison to the time period of the LFO oscillations. The LPF filter eliminates high frequency oscillations. Its time constant $T_{l p f}$ should cause at most 10 degrees lag at the LFO frequency. Note that, the " $\sim$ " character on top of " $d^{2}$ " in (31)-(33) and (35)-(36) means that the second derivative of the VSG frequency is an estimated quantity.

\section{Simulation Results And Discussions}

In this section, the studied power system is firstly described. Then, the proposed scheme is compared against the dual-adaptivity and bang-bang methods explained in Section 3. Finally, the performance of the primary frequency control is discussed for various VSG penetration levels ranged from $25 \%$ to $75 \%$ of the total system generation.

\subsection{Description of the Studied Power System}

The studied two-area system is implemented in DIgSILENT PowerFactory 2019, as depicted in Fig. 7. This benchmark poses the challenge of the LFO damping [30]. The original system data can be found in [9]. The nominal system frequency is $50 \mathrm{~Hz}$. In the VSG $25 \%$ scenario, one of the four SGs is replaced by a VSG labelled VSG4. Parameters of the VSG and its virtual inertia controller are listed in Table 1 and Table 2. The power factor of the VSG unit is 0.85 . All SG and VSG units are equipped with static exciters with high transient gain [9]. A reheat steam turbine and a generic speed governor are used as the VPM and PCO

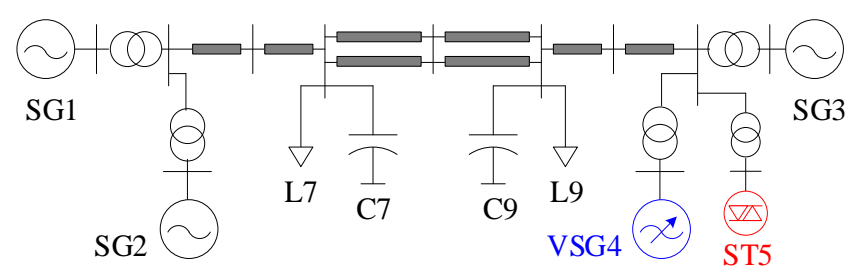

Fig. 7 The single line diagram of the studied two-area system 


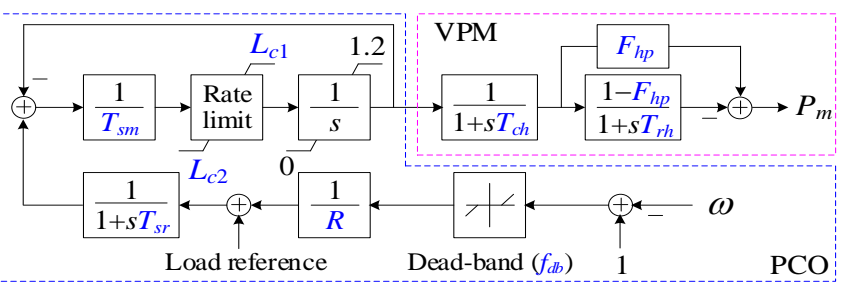

Fig. 8 The steam turbine (VPM) and governing (PCO) systems

Table 1 Virtual Synchronous Generator VSG4 Parameters

\begin{tabular}{cccccccccc}
\hline Parameter & $\boldsymbol{S}$ & $\boldsymbol{H}$ & $\boldsymbol{x}_{\boldsymbol{l}}$ & $\boldsymbol{x}_{\boldsymbol{d}}$ & $\boldsymbol{x}_{\boldsymbol{q}}$ & $\boldsymbol{x}_{\boldsymbol{d}}^{\prime}$ & $\boldsymbol{x}_{\boldsymbol{q}}^{\prime}$ & $\boldsymbol{T}_{\boldsymbol{d} \mathbf{0}}^{\prime}$ & $\boldsymbol{T}_{\boldsymbol{q} \mathbf{0}}^{\prime}$ \\
\hline Unit & MVA & $s$ & p.u. & p.u. & p.u. & p.u. & p.u. & $s$ & $s$ \\
Value & 900 & 6.175 & 0.2 & 1.8 & 1.8 & 0.3 & 0.3 & 8 & 0.4 \\
\hline
\end{tabular}

Table 2 The VSG Virtual Inertia Controller Parameters

\begin{tabular}{|c|c|c|c|c|c|c|c|c|}
\hline arameter & $\boldsymbol{k}_{g}$ & $k_{t p}$ & $\boldsymbol{k}_{t n} \boldsymbol{T}_{a v g}$ & $T_{l p f}$ & $T_{d}$ & $\boldsymbol{k}_{\boldsymbol{r} 1 c}$ & $k_{r 2 c}$ & $\boldsymbol{R}_{\min } \boldsymbol{R}_{\max }$ \\
\hline & & - & & & $s$ & - & & $\mathrm{Hz} / \mathrm{s} \mathrm{Hz} / \mathrm{s}$ \\
\hline Value & 1e13 & 1 & -0.5 & 0.05 & 0.1 & 0.25 & $0.25 \mathrm{C}$ & 0.0250 .25 \\
\hline
\end{tabular}

Table 3 The PCO and VPM Units Parameters [9], [31]

\begin{tabular}{cccccccccc}
\hline Parameter & $\boldsymbol{f}_{\boldsymbol{d} \boldsymbol{b}}$ & $\boldsymbol{R}$ & $\boldsymbol{T}_{\boldsymbol{s r}}$ & $\boldsymbol{T}_{\boldsymbol{s m}}$ & $\boldsymbol{L}_{\boldsymbol{c} \mathbf{1}}$ & $\boldsymbol{L}_{\boldsymbol{c} \mathbf{2}}$ & $\boldsymbol{T}_{\boldsymbol{c h}}$ & $\boldsymbol{T}_{\boldsymbol{r h}}$ & $\boldsymbol{F}_{\boldsymbol{h} \boldsymbol{p}}$ \\
\hline Unit & $\mathrm{mHz}$ & - & $s$ & $s$ & $p . u / s$. & $p . u / s$. & $s$ & $s$ & - \\
Value & 15 & 0.05 & 0.1 & 0.3 & 0.1 & -1 & 0.3 & 7 & 0.3 \\
\hline
\end{tabular}

Table 4 System Power Flow (in \% of base demand $2734 \mathrm{MW}$ ) $\begin{array}{llllllllll}\text { Parameter SG1 } & \text { SG2 } & \text { SG3 } & \text { VSG4 } & \text { ST5 } & \text { L7 } & \text { L9 } & \text { C7 } & \text { C9 }\end{array}$

$\begin{array}{llllllllll}\mathrm{P}(\%) & \text { Slack } & 25.6 & 26.3 & 25.6 & 10.24 & 38.9 & 71.1 & - & -\end{array}$

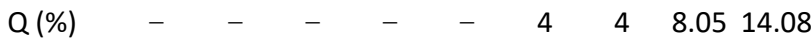

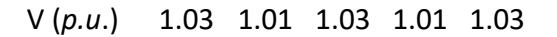

sections for the SGs and VSG plants. However, only the PCO of SG1 and SG3 are in service. Fig. 8 illustrates schematics of the VPM and PCO systems and their parameters are provided in Table 3. To deteriorate the system LFO damping, the PSSs are deliberately deactivated and self-damping of the loads is ignored. In addition, the system demand is increased by $10 \%$ to make the LFO oscillations unstable. The reactive compensations are also increased by $10 \%$. The static generator ST5 is added to the original system to supply the increased load and model a generation loss event. It do not provide inertia. The power flow data are given in Table 4.

\subsection{System Performance under Low VSG Penetration}

In the VSG $25 \%$ scenario, the VSG4 is a single generation unit with adaptive virtual inertia. It is assumed that the virtual inertia can be gone up and down by $100 \%$ and $50 \%$ with respect to its base value, respectively. In the following, the system performance under deploying the proposed scheme are compared with those of the dualadaptivity and the bang-bang strategies, in response to tripping off the generator ST5.

The simulation results of the VSG $25 \%$ scenario are illustrated in Figs. 9 to 14, using a fixed step size $(2.5 \mathrm{~ms})$ RMS simulation. All speed and frequency variables, hereafter, are shown in percentage of the nominal frequency $50 \mathrm{~Hz}$. In addition, to gain a better insight into the results, the power and energy quantities are presented in percentage of the system base demand 2734 MW. Furthermore, the VSG virtual inertia $T_{a g}^{*}$ is per unitized by its base value. The COI frequency deviation and the variations of the VSG frequency with respect to the $\mathrm{COI}$ frequency are depicted in Figs. 9.a and 9.b, respectively. Note that these quantities are obtained by the simulation and thus are not estimated. As
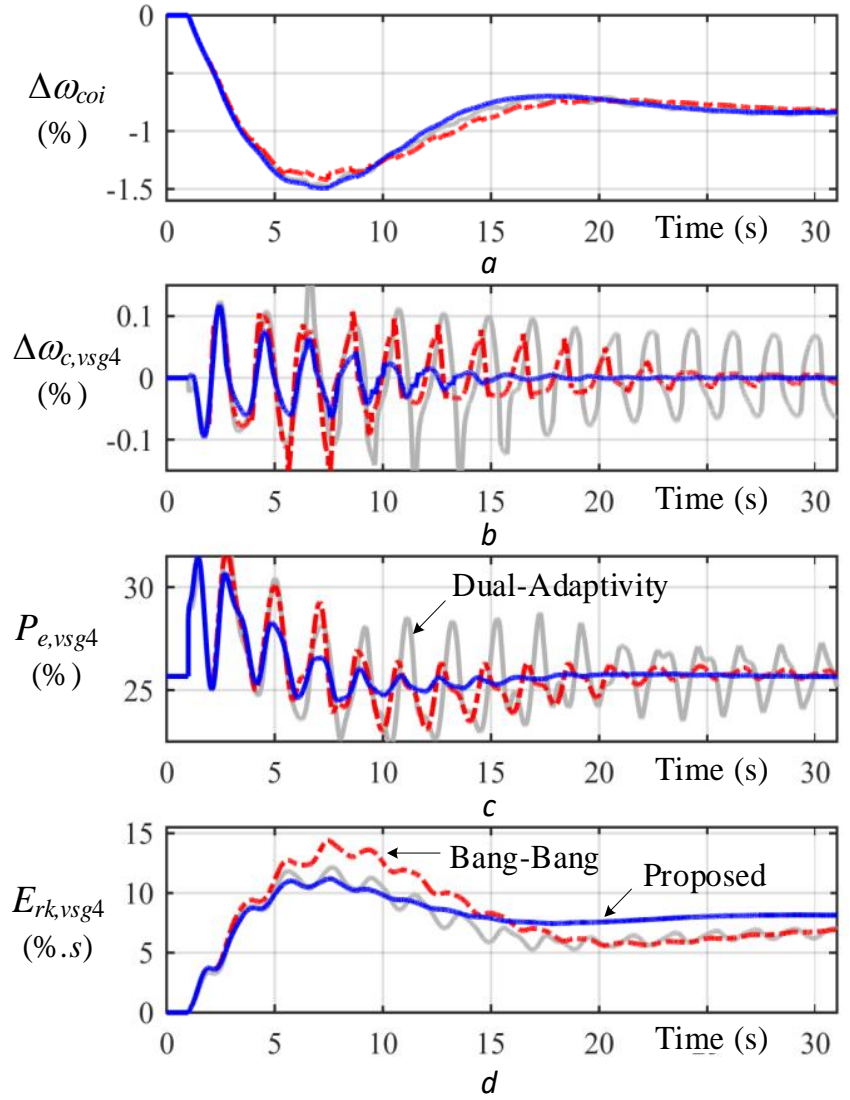

Fig. 9 The simulation results for the VSG $25 \%$ scenario

(a) System COI frequency, (b) Deviation in the VSG4 frequency with respect to system COI frequency, (c) Electrical power of the VSG4, (d) Released kinetic energy by the VSG4 generator

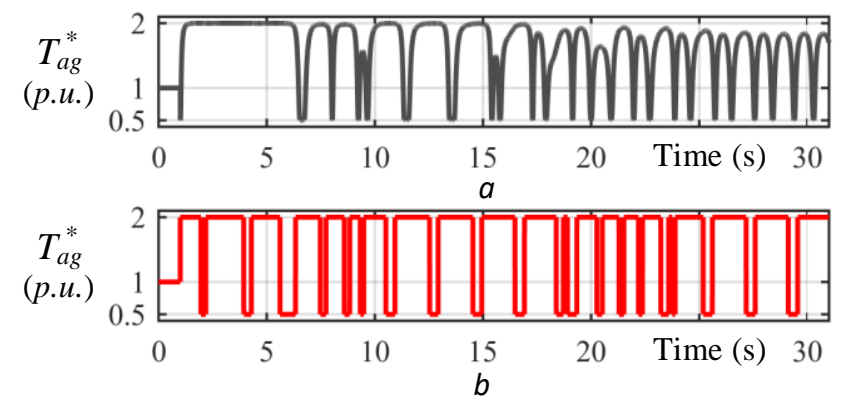

Fig. 10 The VSG4 virtual inertia for the VSG $25 \%$ scenario (a) Dual-adaptivity strategy, (b) Bang-bang strategy

observed, the proposed method provides more oscillations damping than the dual-adaptivity and the bang-bang techniques. This can also be seen in the electrical power quantity portrayed in Fig. 9.c. The amount of the kinetic energy, which is released by the VSG during $t_{1}$ seconds, can be calculated as:

$$
E_{r k}\left(t_{1}\right)=\int_{0}^{t_{1}}\left(P_{e}-P_{m}\right) d t
$$

It is interesting to observe that the suggested technique needs an energy source roughly $22 \%$ smaller than that of the bang-bang method (See Fig. 9.d).

Fig. 10.a shows how the dual-adaptivity method modulates the VSG virtual inertia. Its key parameter $k_{g}$ was found by trial and error to be $1 e 13$. The system experiences lowest oscillations under this value of $k_{g}$. However, the system will be eventually unstable even with this value of $k_{g}$. On the contrary, the bang-bang strategy damps the 
oscillations successfully. The virtual inertia variations are presented in Fig. 10.b, when this approach is used.

In case of the proposed strategy, derivation of the PFC component is described in Fig. 11. The allowable maximum COI RoCoF $R_{\max }$ assumed to be $0.25 \mathrm{~Hz} / \mathrm{s}$ [8]. Also, the parameter $R_{\text {min }}$ mentioned in (28) supposed to be 10 times smaller than the $R_{\max }$. Following the contingency, the PFC component steps up to its upper limit when the rate of the estimated COI frequency exceeds $R_{\min }$. Afterward, it maintains constant until the COI frequency rate reduces again to $R_{\min }$ at the end of the arrest period. On the other hand, the PFC component decreases to its lower limit during the rebound period to achieve a faster frequency recovery.

The LFO component of the VSG4 virtual inertia is adaptively changed as depicted in Fig. 12. In order to explain the underlying concepts behind it, let us consider a zoomed view of Fig. 12, which are displayed in Fig. 13. The variables $k_{r 1}$ and $k_{r 2}$ defined by (31) are also shown. The useless time spans of these variables are covered by the gray areas. At Point A, the VSG frequency is diverging form the COI frequency. Meanwhile, $k_{r 1}$ is declining to zero. Therefore, the LFO component stays on its upper limit to
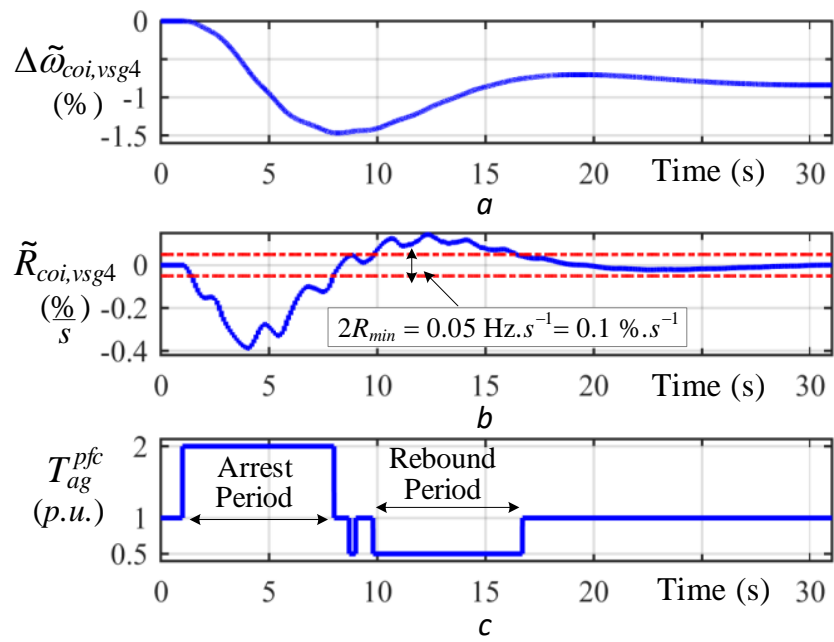

Fig. 11 The VSG4 PFC component in the proposed scheme

(a) Estimated COI frequency by VSG4 using (29), (b) Estimated COI frequency gradient by VSG4 using (30), (c) VSG4 PFC inertia component using (26)
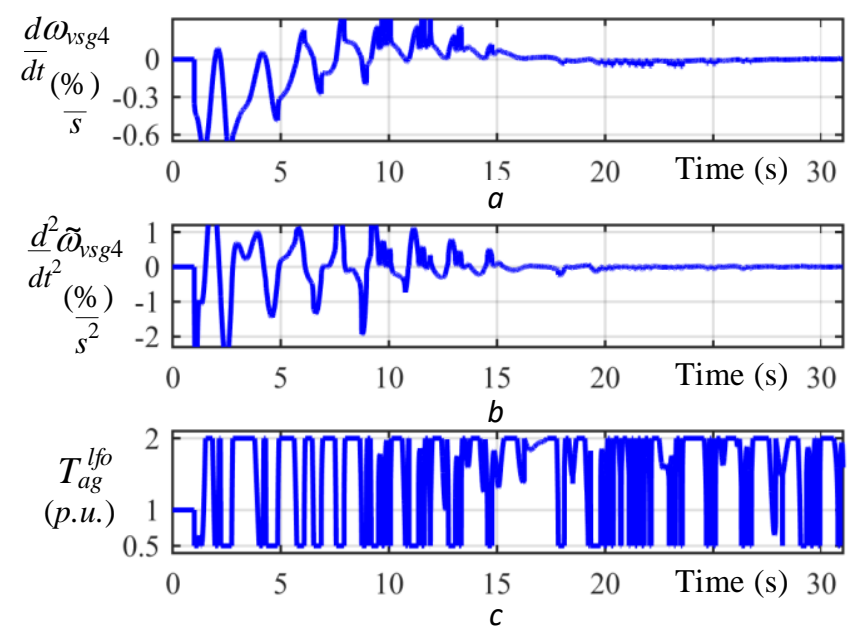

Fig. 12 The VSG4 LFO component in the proposed scheme

(a) Derivative of the VSG4 frequency using (14), (b) Estimated second derivative of the VSG4 frequency using (36), (c) VSG4 LFO inertia component using (33) restrict the frequency deviation. From Point B to Point C, the LFO component drops from its upper limit to its lower limit proportional with $k_{r 1}$. Then, this component persists on its lower limit up to Point $\mathrm{D}$, where $k_{r 2}$ reaches its critical value $k_{r 2 c}$, in order to speed up the frequency recovery. After which, the LFO component returns to its upper limit in proportional with $k_{r 2}$ reduction.

Finally, how the virtual inertia is synthesized by the PFC and LFO components is clarified at Fig. 14. The parameter $k_{p f c}$ along with its constituents $k_{p f c 0}$ and $k_{p f c 1}$ are plotted in Fig. 14.a. It steps up to one and sustains up to 1.0 seconds immediately after the event. Next, it changes in proportional with the absolute value of the estimated COI RoCoF. As stated by (24), this parameter determines the portion of the each components in the virtual inertia. The impact of $k_{p f c}$ on increasing and decreasing of the virtual inertia at the arrest and rebound stages can be clearly seen in Fig. 14.b.

\subsection{System Performance under Various VSG Penetrations}

In the following, the performance of the primary
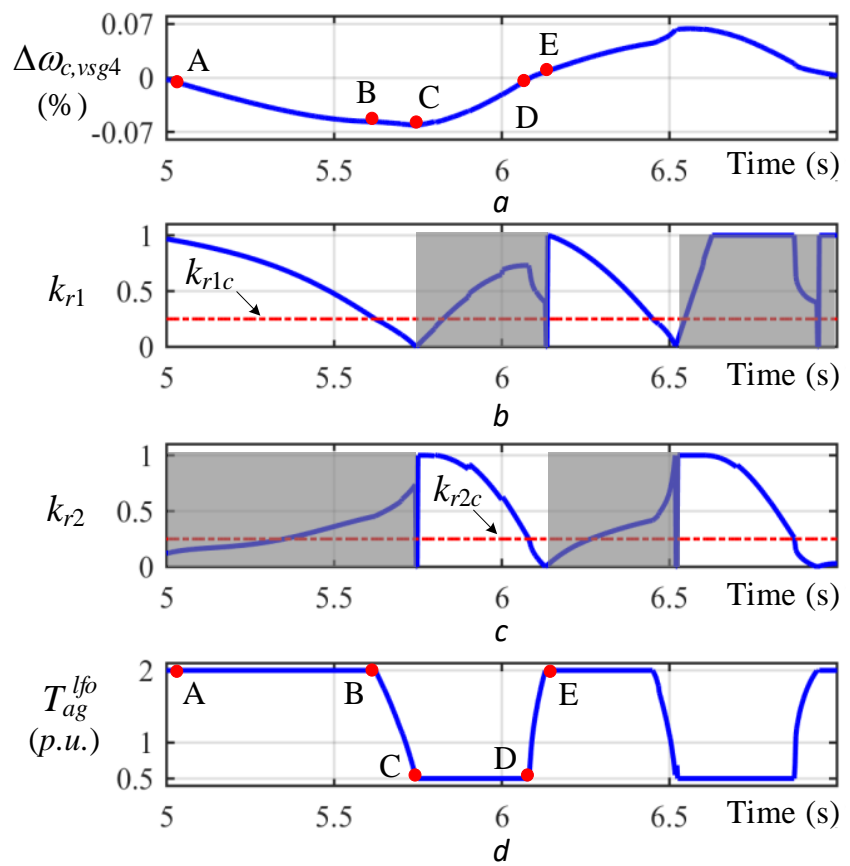

Fig. 13 The zoomed view of Fig. 12

(a) Deviation in the VSG4 frequency with respect to the COI frequency, (b) Auxiliary variable $k_{r 1}$ using (31), (c) Auxiliary variable $k_{r 2}$ using (31), (d) VSG4 LFO inertia component using (33)
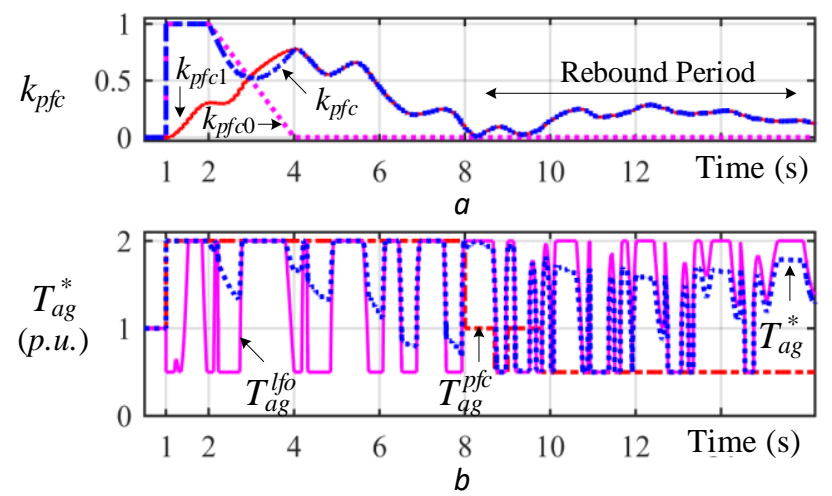

Fig. 14 The VSG4 virtual inertia in the proposed scheme (a) Auxiliary variable $k_{p f c}$ using (34), (b) VSG4 virtual inertia with its PFC and LFO components 
Table 5 Generator Types for the Studied Scenarios

\begin{tabular}{ccccc}
\hline VSG Penetration & \multicolumn{4}{c}{ Generator Number } \\
Level (\%) & 1 & 2 & 3 & 4 \\
\cline { 2 - 5 } 25 & SG & SG & SG & VSG \\
50 & SG & SG & VSG & VSG \\
75 & SG & VSG & VSG & VSG \\
\hline
\end{tabular}

frequency control is investigated under various VSG penetration levels. To this end, three operation scenarios are defined as illustrated in Table 5. The parameters of the VSG units are identical with Table 1 and Table 2. However, the base inertia constant $H$ of each VSGs is equal to the replaced SG generator. The other system information are similar with Table 3 and Table 4. The generators 1 and 3 conduct the PFC in all of the scenarios. Fig. 15 compares the performance of the proposed strategy and that of the bangbang method for different VSG penetration levels. The considered event is tripping out of the ST5. The maximum COI frequency deviation $\Delta \omega_{c o i}^{\max }$ and the frequency recovery time $T_{r e c}$ are defined in Fig. 16. In addition, the $R_{c o i}^{\mathrm{min}}$ denotes the minimum COI RoCoF during 2 seconds following the event, which is calculated with 0.1 second time window. Moreover, the $E_{r k, v s g}^{\max }$ is the maximum total
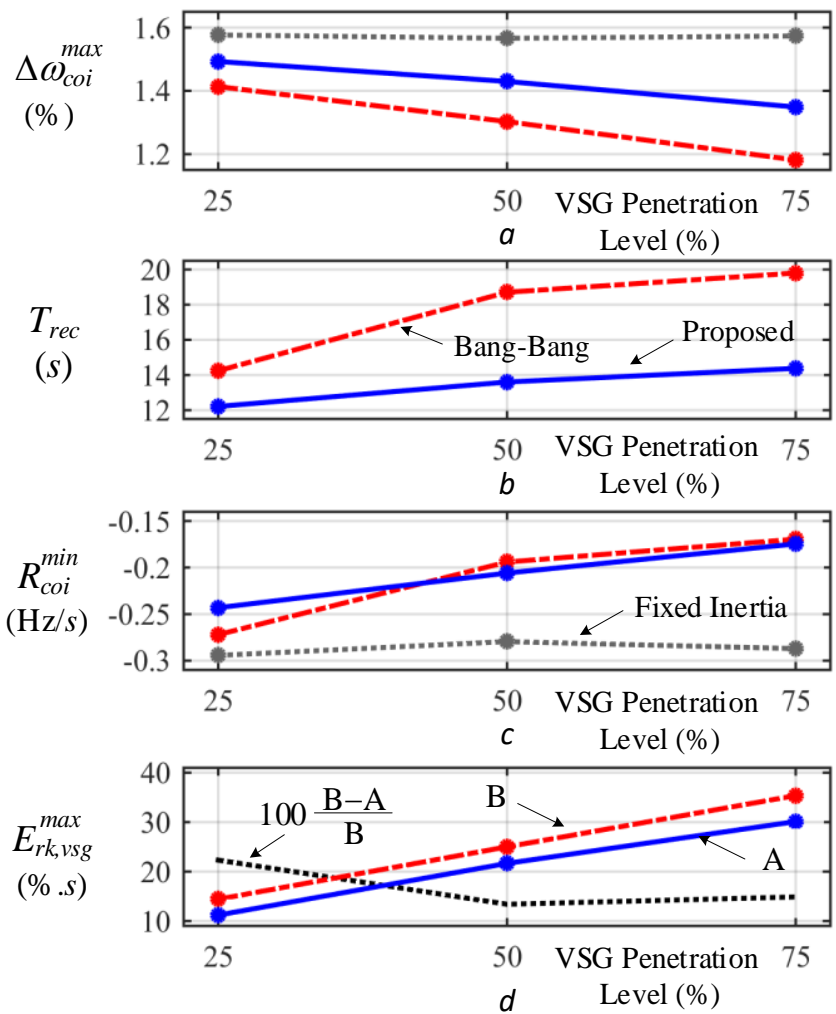

Fig. 15 The primary frequency control performance for the bangbang (dash-dot) and the proposed (solid) methods with different $V S G$ penetration levels

(a) Maximum deviation in the COI frequency, (b) Frequency recovery time, (c) Minimum of the COI frequency gradient, (d) Maximum of the total released kinetic energy by the VSG generators

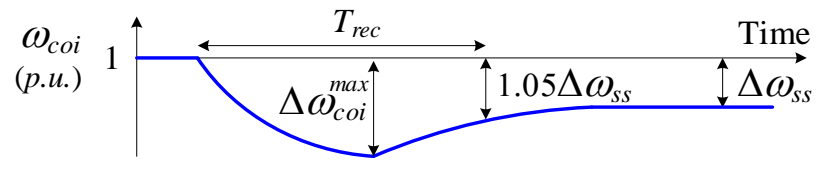

Fig. 16 Definitions for maximum frequency deviation and frequency recovery time measures
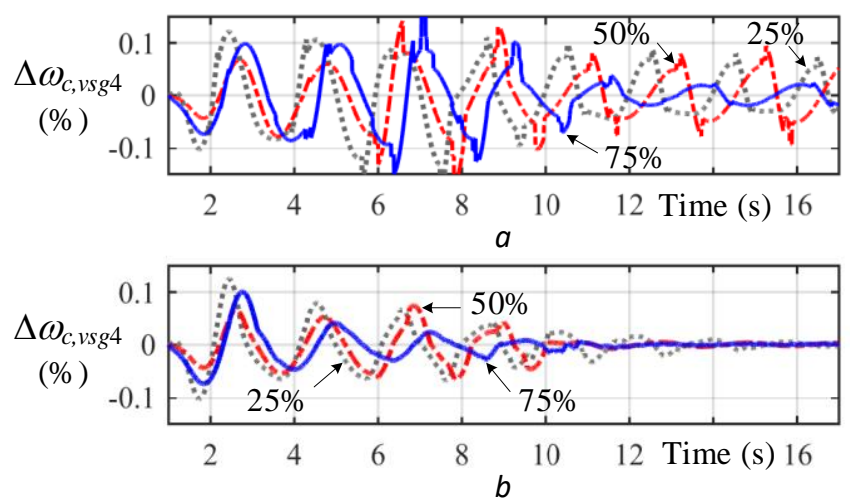

Fig. 17 VSG4 frequency deviation with respect to the COI frequency for different VSG penetration levels

(a) Bang-bang strategy, (b) Proposed strategy
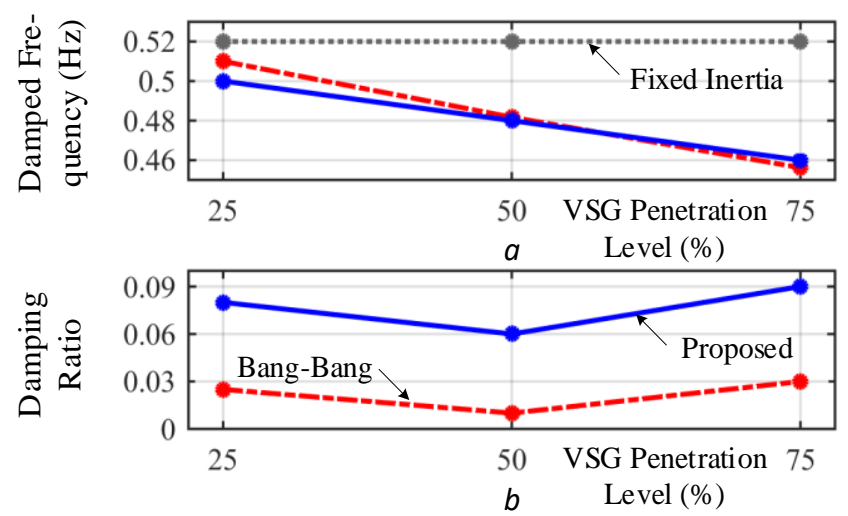

Fig. 18 The damping characteristics of the VSG4 frequency for different VSG penetration levels

(a) Damped frequency, (b) Damping ratio

kinetic energy released by the VSGs following the incident. For the sake of comparison, the results of the Fixed Inertia scenario, in which virtual inertia of the VSGs maintains at 1.0 p.u. following the event, are also shown in Fig. 15. However, the system is not stable in this case. As can be seen, the system frequency is recovered significantly faster once the proposed scheme is deployed. However, the maximum COI frequency deviation in case of the suggested approach is larger than that of the bang-bang strategy. In fact, this superiority of the bang-bang method involves higher cost due to the requirement of larger sources of energy (See Fig. 15.d). The effectiveness of the proposed and the bang-bang techniques in mitigation of the $R_{c o i}^{\min }$ is approximately similar.

Fig. 17 shows deviations of the VSG4 frequency with respect to the $\mathrm{COI}$ frequency for the studied scenarios. It can be clearly seen that the proposed strategy provides more LFO damping. For better comparison, the damped frequency and damping ratio [9] of these traces are provided in Fig. 18. The damped frequency declines with the VSG penetration for both strategies. Although, the proposed scheme provides substantially more damping in comparison to the bang-bang method.

\section{Conclusion}

In this paper, an efficient adaptive strategy is proposed to control the virtual inertia of the virtual synchronous generators (VSGs). This can mitigate primary frequency control (PFC) considering the three proposed optimization 
problems simultaneously. mechanism and provide the electromechanical low frequency oscillations (LFOs) damping. Accordingly, the virtual inertia is synthesized by two parts named PFC and LFO components. The former one is synchronized with the PFC operational time. It rises the virtual inertia during the center-of-inertia (COI) frequency drop period to reduce the rate of change of frequency and decreases it in frequency recovery period to accomplish a faster frequency recovery. The LFO component, which is used to provide LFO damping, is synchronized with the LFO oscillations. It increases the virtual inertia whenever the VSG frequency diverges from the COI frequency and decreases it while the VSG frequency converges to the COI frequency. In this context, the second derivative of the VSG frequency is deployed to estimate the VSG frequency deviation with respect to the COI frequency. The second derivative itself is estimated using the derivatives of the VSG state-space variables and currents of its virtual circuits. The simulation results confirm the superiority of the proposed strategy in the PFC performance mitigation and the LFO damping in comparison with the recently proposed methods in literatures. Furthermore, the proposed approach decreases the required capacity of the VSG energy source by about $15 \%$.

\section{References}

[1] "Nordic Report Future System Inertia". ENTSO-E, Tech. Rep., Jan. 2018.

[2] RG-CE System Protection \& Dynamics Sub Group, "Frequency stability evaluation criteria for the synchronous zone of continental europe," ENTSO-E, Tech. Rep., March 2016.

[3] B. Kroposki et al., "Achieving a 100\% Renewable Grid: Operating Electric Power Systems with Extremely High Levels of Variable Renewable Energy," in IEEE Power and Energy Magazine, vol. 15, no. 2, pp. 61-73, March-April 2017.

[4] F. Milano, F. Dörfler, G. Hug, D. J. Hill and G. Verbič, "Foundations and Challenges of Low-Inertia Systems (Invited Paper)," 2018 Power Systems Computation Conference (PSCC), Dublin, 2018, pp. 1-25.

[5] J. Fang, H. Li, Y. Tang and F. Blaabjerg, "On the inertia of future more-electronics power systems," IEEE J. Emerg. Select. Topics Power Electronics, vol. 7, no. 4, pp. 2130-2146, Dec. 2019.

[6] H. P. Beck and R. Hesse, "Virtual synchronous machine," 9th Int. Conf. Electr. Power Quality Utilisation, Barcelona, pp. 1-6, 2007.

[7] M. Eremia and M. Shahidehpour, Eds., Handbook of Electrical Power System Dynamics: Modeling, Stability, and Control. Wiley-IEEE Press, 2013.

[8] F. Teng and G. Strbac, "Assessment of the Role and Value of Frequency Response Support From Wind Plants," IEEE Trans. Sustain. Energy, vol. 7, no. 2, pp. 586-595, April 2016.

[9] P. Kundur, N. J. Balu, and M. G. Lauby, Power System Stability and Control. New York: McGraw-Hill, 1994.

[10] Q. C. Zhong and G. Weiss, "Synchronverters: Inverters that mimic synchronous generators," IEEE Trans. Ind. Electron., vol. 58, no. 4, pp. 1259-1267, Apr. 2011.

[11] S. Dong and Y. C. Chen, "Adjusting synchronverter dynamic response speed via damping correction loop," IEEE Trans. Energy Convers., vol. 32, no. 2, pp. 608-619, June 2017.

[12] S. Dong and Y. C. Chen, "A method to directly compute synchronverter parameters for desired dynamic response," IEEE Trans. Energy Convers., vol. 33, no. 2, pp. 814-825, Jun. 2018.

[13] M. Guan, W. Pan, J. Zhang, Q. Hao, J. Cheng and X. Zheng, "Synchronous generator emulation control strategy for voltage source converter (VSC) stations," IEEE Trans. Power Syst., vol. 30, no. 6, pp. 3093-3101, Nov. 2015.

[14] L. Huang, H. Xin and Z. Wang, "Damping low-frequency oscillations through VSC-HVdc stations operated as virtual synchronous machines," IEEE Trans. Power Electron., vol. 34, no. 6, pp. 5803-5818, June 2019.

[15] M. Ebrahimi, S. A. Khajehoddin and M. Karimi-Ghartemani, "An improved damping method for virtual synchronous machines,"
IEEE Trans. Sustain. Energy, vol. 10, no. 3, pp. 1491-1500, Jul. 2019.

[16] W. Du, Q. Fu and H. F. Wang, "Power system small-signal angular stability affected by virtual synchronous generators," IEEE Trans. Power Syst., vol. 34, no. 4, pp. 3209-3219, Jul. 2019.

[17] T. Shintai, Y. Miura and T. Ise, "Oscillation damping of a distributed generator using a virtual synchronous generator," IEEE Trans. Power Deliv., vol. 29, no. 2, pp. 668-676, Apr. 2014.

[18] J. Alipoor, Y. Miura and T. Ise, "Power system stabilization using virtual synchronous generator with alternating moment of inertia," IEEE J. Emerg. Select. Topics Power Electronics, vol. 3, no. 2, pp. 451-458, Jun. 2015

[19] D. Li, Q. Zhu, S. Lin and X. Y. Bian, "A Self-adaptive inertia and damping combination control of VSG to support frequency stability," IEEE Trans. Energy Convers., vol. 32, no. 1, pp. 397398, Mar. 2017

[20] F. Wang, L. Zhang, X. Feng and H. Guo, "An adaptive control strategy for virtual synchronous generator," IEEE Trans. Ind. Appl., vol. 54, no. 5, pp. 5124-5133, Sept.-Oct. 2018.

[21] X. Hou, Y. Sun, X. Zhang, J. Lu, P. Wang and J. M. Guerrero, "Improvement of frequency regulation in VSG-Based AC microgrid via adaptive virtual inertia," IEEE Trans. Power Electron., vol. 35, no. 2, pp. 1589-1602, Feb. 2020.

[22] M. Li, W. Huang, T. NengLing, L. Yang, D. Duan and Z. Ma, “A dual-adaptivity inertia control strategy for virtual synchronous generator," IEEE Trans. Power Syst., vol. 35, no. 1, pp. 594-604, Jan. 2020

[23] Y. Cao, W. Wang, Y. Li, Y. Tan, C. Chen, L. He, U. Häger, and C. Rehtanz, "A virtual synchronous generator control strategy for VSC-MTDC systems," IEEE Trans. Energy Convers., vol. 33, no. 2, pp. 750-761, Jun. 2018.

[24] E. Rakhshani, D. Remon, A. M. Cantarellas, J. M. Garcia and P. Rodriguez, "Virtual synchronous power strategy for multiple HVDC interconnections of multi-area AGC power systems," IEEE Trans. Power Syst., vol. 32, no. 3, pp. 1665-1677, May 2017.

[25] A. Fathi, Q. Shafiee and H. Bevrani, "Robust frequency control of microgrids using an extended virtual synchronous generator," IEEE Trans. Power Syst., vol. 33, no. 6, pp. 6289-6297, Nov. 2018.

[26] H. Alrajhi Alsiraji and R. El-Shatshat, "Comprehensive assessment of virtual synchronous machine based voltage source converter controllers," in IET Gener. Transm. Distrib., vol. 11, no. 7, pp. 1762-1769, 1152017.

[27] Krause, P., Wasynczuk, O., Sudhoff, S.D. and Pekarek, S., Analysis of electric machinery and drive systems, John Wiley \& Sons, 2013.

[28] DIgSILENT GmbH, "DIgSILENT PowerFactory 2019 Synchronous Machine Technical Reference Documentation," Tech. Rep., July 22, 2019.

[29] Industrial Manual, Siemens "Numerical Voltage, Frequency, and Overflux Protection Relay SIPROTEC 7RW600 V1.0 Instruction Manual", Available Online: https://www.downloads.siemens.com/download-center/.

[30] C. Canizares et al., "Benchmark models for the analysis and control of small-signal oscillatory dynamics in power systems," IEEE Trans. Power Syst., vol. 32, no. 1, pp. 715-722, Jan. 2017.

[31] P. M. Anderson and M. Mirheydar, "A low-order system frequency response model," IEEE Trans. Power Syst., vol. 5, no. 3, pp. 720-729, Aug. 1990 . 